\title{
Refocusing on hypertension control in Canada
}

\author{
Alexander A. Leung MD MPH, Alan Bell MD, Ross T. Tsuyuki PharmD MSc, Norman R.C. Campbell MD
}

Cite as: CMAJ 2021 June 7;193:E854-5. doi: 10.1503/cmaj.210140

anada has been an international leader in hypertension care, but this appears to be changing. After more than 60 years of declining rates of cardiovascular death, trends are now reversing, along with reduced rates of detection, treatment and control of hypertension. ${ }^{1-3}$ According to a survey conducted in 2007-2009, 82\% of Canadians with hypertension were treated and $69 \%$ were controlled; by comparison, survey data from $2016-17$ showed that only $72 \%$ of affected adults were treated and a mere $58 \%$ were adequately controlled. ${ }^{2}$ In the most recent cycle of a national survey, fewer than two-thirds of women reported being treated for hypertension and fewer than half were controlled for hypertension. ${ }^{2}$ During the same period, clinical practice guidelines from Hypertension Canada became more intensive, with lower recommended targets for systolic blood pressure in selected high-risk patients with no distinctions between women and men with respect to treatment indications or blood pressure goals. Although the specific reasons underlying the changes in national hypertension treatment and control are unknown, we suggest several plausible explanations, including the loss of federal government support for hypertension surveillance, waning emphasis on implementation and evaluation of hypertension guidelines and termination of industry sponsorship for education initiatives as generic medications became more widely used. ${ }^{2}$

Recent controversies and discrepancies about optimal blood pressure targets, perceived risks of treatment and expert recommendations may have dissuaded some clinicians from treating hypertension in certain patients, especially older adults. However, ample data support the benefits and safety of hypertension control in this population. For instance, in the Hypertension in the Very Elderly Trial, treatment of older adults (mean age $83.6 \mathrm{yr} ; 60.5 \%$ female) with a baseline systolic blood pressure of $160 \mathrm{~mm} \mathrm{Hg}$ or higher resulted in a mean blood pressure reduction of 15.0/6.1 mm Hg compared with placebo. The treatment group also had a $3.0 \%$ absolute risk reduction in any cardiovascular event $(7.1 \%$ v. $10.1 \%$ for placebo, number needed to treat $33, p<0.001)$ and a $2.2 \%$ absolute risk reduction in all-cause death (10.1\% v. $12.3 \%$, number needed to treat $46, p=0.02)$ over a median of only 1.8 years. ${ }^{4}$ Moreover, several trials have shown the mortality benefit of reducing blood pressure in older patients, irrespective of frailty (excluding patients with limited life expectancy or dementia, or those needing institutionalized care). ${ }^{4,5}$ Overall, rates of adverse effects from treatment are low. ${ }^{4,5}$ Indeed, treatment of hypertension is supported by high-

\section{KEY POINTS}

- Treatment of hypertension is highly effective for reducing cardiovascular morbidity, preventable disability and death across age groups.

- National rates of hypertension treatment and control have worsened in the last decade.

- Hypertension control should again be made a national priority, with optimization of clinical care using global best practices and re-engagement with government.

quality evidence for patients across a broad range of cardiovascular risk profiles and age groups for both men and women. ${ }^{6,7}$

The determinants of blood pressure control are admittedly complex, but innovative, multidimensional strategies can improve management and outcomes. Specifically, a combination of health system, provider-level and patient-level approaches has proven highly effective in closing care gaps and is generalizable to many settings. ${ }^{8}$ The World Health Organization and its partners developed the HEARTS program that outlines best practices for improving hypertension management to reduce cardiovascular disease. ${ }^{9}$ These include screening all adults, identifying associated health risks, promoting team-based care, using a treat-to-target approach with simple directive algorithms and monitoring performance using registries with reporting functions. ${ }^{9}$ Applying these standards, Kaiser Permanente Northern California reported an impressive rise in hypertension control, from $44 \%$ in 2001 to $90 \%$ in $2015,{ }^{10}$ with similar successes observed in other jurisdictions following implementation. ${ }^{8}$ Adapting these principles of best practice into routine care is pivotal to achieving improvements in hypertension control.

In Canada, national priorities should include engagement with front-line primary care to provide universal screening for high blood pressure at routine clinical encounters with adults. Comprehensive care should be team-based. Partnerships with community, primary care and pharmacy programs for screening and management may serve people with hypertension who do not regularly access health care. For most patients who require treatment, target levels for blood pressure should be less than $140 / 90 \mathrm{~mm} \mathrm{Hg}$ (or $130 / 80 \mathrm{~mm} \mathrm{Hg}$, for those with diabetes). To achieve this goal, the adoption of standardized treatment algorithms, particularly those that also incorporate single pill, 
fixed-dose combinations of antihypertensive drugs, can help with physicians' therapeutic inertia and patients' ability to adhere to medication, simplify guideline recommendations and provide guidance for specific drugs, doses and titration schedules (e.g., the World Health Organization's Resolve to Save Lives toolkit, https://linkscommunity.org/toolkit/hypertension -control).9,11 Treatment algorithms can be adapted in consultation with local experts and modified based on common formularies. ${ }^{8}$ Primary care providers can still give individualized care, particularly for patients with compelling indications for alternative therapy or a history of adverse effects, but standardized management protocols are effective and safe for most people. ${ }^{8,10}$ Finally, registries with regular performance reporting at the provider, clinic and regional level should be used to maintain best practices, to provide audit data and feedback for learning, to offer incentives and to close important care gaps. ${ }^{9}$

Canada is now facing its lowest rates of hypertension treatment and control in more than a decade, and we cannot afford to remain complacent. With an aging population, the number of people living with hypertension is growing, and the number of adults with hypertension who remain untreated or uncontrolled will only increase if we do not act, which will lead to greater rates of preventable disability and death. Renewed interest in blood pressure control is needed, with resurrection of previous implementation efforts to provide effective care and re-engagement of the federal government with health and scientific sectors to enhance monitoring and evaluation.

\section{References}

1. Joffres M, Falaschetti E, Gillespie C, et al. Hypertension prevalence, awareness, treatment and control in national surveys from England, the USA and Canada, and correlation with stroke and ischaemic heart disease mortality: a cross-sectional study. BMJ Open 2013;3:e003423.

2. Leung AA, Williams JVA, McAlister FA, et al. Worsening hypertension awareness, treatment, and control rates in Canadian women between 2007 and 2017. Can J Cardiol 2020;36:732-9.

3. Global burden of disease study. Seattle (WA): University of Washington, Institute for Health Metrics and Evaluation; 2017. Available: http://vizhub. healthdata.org/gbd-compare (accessed 2021 Jan. 19).

4. Beckett NS, Peters R, Fletcher AE, et al. Treatment of hypertension in patients 80 years of age or older. N Engl J Med 2008;358:1887-98.

5. Williamson JD, Supiano MA, Applegate WB, et al. Intensive vs standard blood pressure control and cardiovascular disease outcomes in adults aged $\geq 75$ years: a randomized clinical trial. JAMA 2016;315:2673-82.

6. Blood Pressure Lowering Treatment Trialists' Collaboration. Blood pressurelowering treatment based on cardiovascular risk: a meta-analysis of individual patient data. Lancet 2014;384:591-8.

7. Xie X, Atkins E, Lv J, et al. Effects of intensive blood pressure lowering on cardiovascular and renal outcomes: updated systematic review and meta-analysis. Lancet 2016;387:435-43.

8. Fontil V, Gupta R, Moise N, et al. Adapting and evaluating a health system intervention from Kaiser Permanente to improve hypertension management and control in a large network of safety-net clinics. Circ Cardiovasc Qual Outcomes 2018;11:e004386.

9. HEARTS technical package for cardiovascular disease management in primary health care: implementation guide. Geneva: World Health Organization; 2018.

10. Jaffe MG, Young JD. The Kaiser Permanente Northern California Story: improving hypertension control from $44 \%$ to $90 \%$ in 13 years (2000 to 2013). J Clin Hypertens (Greenwich) 2016;18:260-1.

11. Jaffe MG, Frieden TR, Campbell NRC, et al. Recommended treatment protocols to improve management of hypertension globally: a statement by Resolve to Save Lives and the World Hypertension League (WHL). J Clin Hypertens (Greenwich) 2018;20:829-36.
Competing interests: Alexander Leung was involved in the development of the Hypertension Canada clinical practice guidelines (2016-2020) and is cochair of Hypertension Canada's research and evaluation committee. He also reports grants from the Canadian Institutes of Health Research and Hypertension Canada, outside the submitted work. Alan Bell is a member of the board of directors of Hypertension Canada, cochair of the implementation and education committee (2016-present) and coauthor of the clinical practice guidelines (2020). Ross Tsuyuki is the president of Hypertension Canada (2020present), cochair of the implementation and education committee (2017-present), member of the Implementation Research Task Force (2005-2011) and the Public Recommendations Task Force (2007-2010), and editor-in-chief of the Canadian Pharmacists Journal. He also reports consulting fees from Emergent Biosolutions, Shoppers Drug Mart and HLS Therapeutics, as well as an honorarium from Sanofi Genzyme. Norman Campbell is past president and special advisor to the board of the World Hypertension League, and is vice chair of the Canadian Hypertension Advisory Committee. He reports support from Resolve to Save Lives, World Bank, the Pan American Health Organization and the World Health Organization related to involvement in hypertension control programs. He is also a consultant to the Hearts in the Americas Initiative of the Pan American Health Organization (2012present), and a reviewer of the World Health Organization Hearts Program (2014-present). No other competing interests were declared.

This article has been peer reviewed.

Affiliations: Department of Medicine (Leung, Campbell) and of Community Health Sciences (Leung, Campbell), University of Calgary, Calgary, Alta.; Department of Family and Community Medicine (Bell), University of Toronto, Toronto, Ont.; Department of Pharmacology
(Tsuyuki) and of Medicine (Tsuyuki), University of Alberta, Edmonton, Alta.

Contributors: All authors contributed to the conception and design of the work. Alexander Leung drafted the manuscript. All authors revised it critically for important intellectual content, gave final approval of the version to be published and agreed to be accountable for all aspects of the work.

Content licence: This is an Open Access article distributed in accordance with the terms of the Creative Commons Attribution (CC BY-NC-ND 4.0) licence, which permits use, distribution and reproduction in any medium, provided that the original publication is properly cited, the use is noncommercial (i.e., research or educational use), and no modifications or adaptations are made. See: https://creativecommons. org/licenses/by-nc-nd/4.0/

Correspondence to: Alexander Leung, aacleung@ucalgary.ca 